\title{
How to define the relative contraindications to oral anticoagulant therapy
}

\author{
Roberto F.E. Pedretti \\ Department of Cardiac Rehabilitation, Istituti Clinici Scientifici Maugeri, IRCCS Tradate, Italy
}

\begin{abstract}
There is currently a lack of consensus on which anticoagulant therapy contraindications should be considered "absolute" and which should be considered "relative". Guidelines do not clearly identify absolute and relative contraindications to anticoagulant therapy.

Recent guidelines on AF of the European Society of Cardiology underline the relevance of several factors and their use in scores, leaving anyway space to the clinical judgment of the physician. A high bleeding risk score should generally not result per se in a contraindication to anticoagulant therapy. Rather, bleeding risk factors should be identified and treatable factors corrected.

A combined use of a more hierarchical classification of the different bleeding risk factors and the risk scores probably represents the best approach to maximize the benefit of anticoagulant therapy in various clinical settings.
\end{abstract}

Anticoagulation therapy applies to several clinical conditions, i.e. atrial fibrillation (AF), deep venous thrombosis (DVT) and venous thromboembolism (VTE), ischemic heart disease, left ventricle thrombosis. To the need for simplification, most of information reported in the present paper refer to two main clinical setting, AF and DVT; nevertheless, most of observations may be generalized also to different clinical scenarios.

It is well known that oral anticoagulants (OACs) reduce the risk of thromboembolic events associated with AF [1,2], yet evidence suggests that many high-risk patients with $\mathrm{AF}$ are not taking OACs [3]. Underuse of OACs is often attributed to treatment contraindications that create an unfavorable risk/benefit profile for OAC therapy. Evidence

Corresponding author: Roberto F.E. Pedretti, Department of Cardiac Rehabilitation, Istituti Clinici Scientifici Maugeri, IRCCS Tradate, Via Roncaccio 16, 21049, Tradate (VA), Italy. Tel: +39.0331 .829300$ Fax: +39.0331.829555. E-mail: roberto.pedretti@icsmaugeri.it

Key words: Contraindications; atrial fibrillation; oral anticoagulation bleeding.

Received for publication: 22 January 2017

Accepted for publication: 31 January 2017

CC Copyright R.F.E. Pedretti, 2017

Tipografia PI-ME Editrice, Italy

Monaldi Archives for Chest Disease 2017; 87:847

doi: 10.4081/monaldi.2017.847

This article is distributed under the terms of the Creative Commons Attribution Noncommercial License (by-nc 4.0) which permits any noncommercial use, distribution, and reproduction in any medium, provided the original author(s) and source are credited. suggests that old age, language abilities, race, and patient-related and provider-related reasons may determine which patients receive OACs [4,5]. Furthermore, perceived OAC contraindications are highly variable and often differ from one provider to another. Recent evidence suggests that anticoagulation treatment decisions are more likely to be driven by perceived risks such as adverse bleeding [6,7].

There is currently a lack of consensus on which OAC contraindications should be considered "absolute" and which should be considered "relative." The Medical Services Commission of British Columbia has published a set of absolute contraindications to warfarin therapy, including the presence of severe or active bleeding diathesis, nonadherence to medication or INR monitoring, pregnancy, and allergy or intolerance to warfarin [8]. The same set of guidelines lists a set of relative contraindications, including uncontrolled hypertension, severe liver disease, and recent surgery involving the nervous system, spine, or eye, several of which have been reported as absolute contraindications in other studies [8].

On the other hand, guidelines do not clearly identify absolute and relative contraindications to anticoagulant therapy. Recent guidelines on AF of the European Society of Cardiology underline the relevance of several factors and their combined use in scores [9], leaving anyway space to the clinical judgment of the physician.

Assessments of stroke and bleeding risks are essential to selecting oral anticoagulation in patients with AF. Steinberg et al. [10] evaluated outcomes according to physician assessed risk, with comparison to empirical risk scores in a prospective, observational study of 9715 outpatients with AF enrolled in ORBIT-AF, a US national registry.

Stroke and bleeding risks were quantified by physician assignment, $\mathrm{CHADS}_{2}$ and $\mathrm{CHA}_{2} \mathrm{DS}_{2}$-VASc stroke scores, and ATRIA and HAS-BLED bleeding scores. Outcomes were stroke or systemic embolism and major bleeding during a median follow-up of 28 months.

Physician-assessed risk categories did not add significantly to empirical risk scores, in Cox models for outcomes. These data support the use of empirical scores for stroke and bleeding risk stratification, and the need for novel approaches to risk stratification in this population.

ESC guidelines [9] classify bleeding risk factors in 4 main groups: a) modifiable bleeding risk factors

b) potentially modifiable bleeding risk factors

c) non-modifiable bleeding risk factors

d) biomarker-based bleeding risk factors

Several bleeding risk scores have been developed, mainly in patients on vitamin $\mathrm{K}$ antagonists. These include HAS-BLED [hypertension, abnormal renal/liver function (1 point each), stroke, bleeding history or predisposition, labile INR, elderly ( $>65$ years), drugs/alcohol concomitantly (1 point each)], ORBIT (Outcomes Registry for Better Informed Treatment of Atrial Fibrillation), and more recently, the ABC (age, biomarkers, clinical history) bleeding score, which also makes use of selected biomarkers [11-13].

Stroke and bleeding risk factors overlap. For example, older age is one of the most important predictors of both ischaemic stroke and bleeding in AF patients [14,15]. 
Guidelines underline that a high bleeding risk score should generally not result per se in a contraindication to OAC. Rather, bleeding risk factors should be identified and treatable factors corrected [9].

However, tools to assess bleeding risk in patients taking oral anticoagulants, including the HAS-BLED bleeding risk score, may lead to imprecise estimates in the individual patient.

One problem with the bleeding risk scores is that they were developed from studies that included bleeds of differing severity. While any bleed can lead to death or severe disability, they usually do not. The major exception to this is intracranial hemorrhage (ICH). For the purposes of estimating bleeding risk, we think that most patients care deeply about ICH risk and to a lesser extent about epistaxis or gastrointestinal bleeding requiring hospitalization and possibly transfusion. The former (ICH) can be equated in severity to an ischemic stroke while the latter cannot.

One of the more controversial issues is the use of anticoagulants in cancer [16]. Cancer patients are at high risk for venous thromboembolism (VTE), which results in substantial morbidity and mortality. The benefit of perioperative anticoagulant prophylaxis following cancer surgery is well established. However, the risk-benefit trade-offs in non-surgical hospitalized cancer patients and among outpatients receiving chemotherapy are more complex. Emerging evidence suggests that the use of low molecular weight heparin (LMWH) may confer a small survival benefit in cancer patients without VTE. However, specific patient populations that may derive the most benefit have yet to be defined.

A more scholastic classification of the several bleeding risk factors into clinical conditions that may be considered absolute or relative contraindications to OACs may be still useful.

As reported in a Buckinghamshire NHS Trust relative and absolute contraindications to OACS are the following [17]:

\section{Absolute contraindications}

- Known large oesophageal varices.

- Significant thrombocytopenia (platelet count $<50 \times 109 / \mathrm{L}$ ).

- Within 72 hours of major surgery with risk of severe bleeding (defer and reassess the risk postoperatively).

- Previously documented hypersensitivity to either the drug or excipients .

- Acute clinically significant bleed (defer and reassess stroke vs bleeding risk within 3 months).

- Decompensated liver disease or deranged baseline clotting screen (INR $>1.5)$

- Pregnancy or within 48 hours post-partum.

- Severe renal impairment (GFR $<30 \mathrm{~mL} / \mathrm{min} / 1.73 \mathrm{~m}^{2}$ or on dialysis) (contraindication applies for some novel oral anticoagulants).

\section{Relative contraindications}

- Previous history intracranial haemorrhage (as some AF patients, especially those considered at higher stroke risk (i.e. $\mathrm{CHADS}_{2}$ score $\geq 3$ ) may benefit form anti-thrombotic therapy, seek the opinion of a stroke specialist).

- Recent major extracranial bleed within the last 6 months where the cause has not been identified or treated (decision for oral antithrombotic therapy should be deferred).

- Recent documented peptic ulcer within last 3 months (decision for oral antithrombotic therapy should be deferred until treatment for peptic ulcer is completed. In all cases to patients with history of peptic ulcer a pump protonic inhibitor should be prescribed whilst on OACs).
- Recent history of recurrent iatrogenic falls in patient at higher bleeding risk (HAS-BLED $\geq 3$ ). It is important to underline that a risk of falls is not a contraindication to initiate a therapy with OACs (e.g. a patient with an annual stroke risk of $5 \%\left(\mathrm{CHADS}_{2}\right.$ score 2-3) would need to fall 295 times to outweigh stroke reduction benefit of warfarin)

- Dementia or marked cognitive impairment with poor medicines compliance \& no access to caregiver support.

- Chronic alcohol abuse - especially if associated with binge drinking

There is currently a lack of consensus on which OAC contraindications should be considered "absolute" and which should be considered "relative".

Guidelines do not clearly identify absolute and relative contraindications to anticoagulant therapy. Recent guidelines on AF of the European Society of Cardiology underline the relevance of several factors and their combined use in scores [9], leaving anyway space to the clinical judgment of the physician.

The combined use of a more hierarchical classification of the different bleeding risk factors and the risk scores probably represents the best approach to maximize the benefit of anticoagulant therapy in various clinical settings.

\section{References}

1. Wolf PA, Abbott RD, Kannel WB. Atrial fibrillation as an independent risk factor for stroke: the Framingham Study. Stroke 1991; 22:983-8.

2. Hart RG, Pearce LA, Aguilar MI. Meta-analysis: antithrombotic therapy to prevent stroke in patients who have nonvalvular atrial fibrillation. Ann Intern Med 2007;146:857-67.

3. Ogilvie IM, Newton N, Welner SA, et al. Underuse of oral anticoagulants in atrial fibrillation: a systematic review. Am J Med 2010; 123:638-45 e634.

4. Waldo AL, Becker RC, Tapson VF, et al. Hospitalized patients with atrial fibrillation and a high risk of stroke are not being provided with adequate anticoagulation. J Am Coll Cardiol 2005;46:1729-36.

5. Tulner LR, Van Campen JP, Kuper IM, et al. Reasons for undertreatment with oral anticoagulants in frail geriatric outpatients with atrial fibrillation: a prospective, descriptive study. Drugs Aging 2010;27:39-50.

6. Devereaux PJ, Anderson DR, Gardner MJ, et al. Differences between perspectives of physicians and patients on anticoagulation in patients with atrial fibrillation: observational study. BMJ 2001; 323:1218-22.

7. Gross CP, Vogel EW, Dhond AJ, et al. Factors influencing physicians' reported use of anticoagulation therapy in nonvalvular atrial fibrillation: a cross-sectional survey. Clin Ther 2003;25:1750-64.

8. BCGuidelines.ca. Warfarin therapy management. Available from: http://www2.gov.bc.ca/gov/content/health/practitioner-professionalresources/bc-guidelines/warfarin-therapy

9. Kirchhof P, Benussi S, Kotecha D, et al. 2016 ESC Guidelines for the management of atrial fibrillation developed in collaboration with EACTS. The Task Force for the management of atrial fibrillation of the European Society of Cardiology (ESC). Eur Heart J 2016; 37:2893-962.

10. Steinberg BA, Greiner M, Hammill BG, et al. Contraindications to anticoagulation therapy and eligibility for novel anticoagulants in older patients with atrial fibrillation. Cardiovasc Ther 2015;33:177-83.

11. Gage BF, Yan Y, Milligan PE, et al. Clinical classification schemes for predicting hemorrhage: results from the National Registry of Atrial Fibrillation (NRAF). Am Heart J 2006;151:713-9. 
12. Pisters R, Lane DA, Nieuwlaat R, et al. A novel user- friendly score (HAS-BLED) to assess 1-year risk of major bleeding in patients with atrial fibrillation: the Euro Heart Survey. Chest 2010;138: 1093-100.

13. Fang MC, Go AS, Chang Y, et al. A new risk scheme to predict warfarin-associated hemorrhage: The ATRIA (Anticoagulation and Risk Factors in Atrial Fibrillation) Study. J Am Coll Cardiol 2011;58: 395-401.

14. Friberg L, Rosenqvist M, Lip GY. Evaluation of risk stratification schemes for is- chaemic stroke and bleeding in 182678 patients with atrial fibrillation: the Swedish Atrial Fibrillation cohort study. Eur Heart J 2012;33:1500-10.

15. Hijazi Z, Oldgren J, Lindback J, et al. The novel biomarker-based $\mathrm{ABC}$ (age, biomarkers, clinical history)-bleeding risk score for patients with atrial fibrillation: a derivation and validation study. Lancet 2016;387:2302-11.

16. Siegal DM, Garcia D. Anticoagulants in cancer. J Thromb Haemost 2012;10:2230-41.

17. [Internet]. http://www.bucksformulary.nhs.uk/docs/ContraindicationsOralAnticoag\&Anti-plateletsAFPrimaryCare.pdf 